\title{
Modelagem 3-D da Deformaç“o Flexural da Litosfera na Margem Continental do Brasil: Implicações na História de SubsidOrncia das Bacias Marginais
}

Victor Sacek*, Naomi Ussami, Instituto de Geofísica, Astronomia e Ci@Encias AtmosfØricas

Copyright 2006, SBGf - Sociedade Brasileira de Geofísica

Este texto foi preparado para a apresentação no ll Simpósio de Geofísica da Sociedade Brasileira de Geofísica, Natal, 21 -23 de setembro de 2006. Seu conteúdo foi revisado pela Comissão Tecno -científica do II SR-SBGf mas não necessar iamente Representa a opinião da SBGf ou de seus associados. E proibida a reprodução total ou arcial deste material para propósitos comerciais sem prévia autorização da SBGf.p

\section{Resumo}

O objetivo deste projeto foi de obter a soluç"o numØrica pelo MØtodo dos Elementos Finitos (Finite Element Method: FEM) do campo de deformações da placa el/stica fina que representa a Placa da AmØrica do Sul devido às variações temporais e geogr Æ ficas das cargas sedimentares que preencheram as bacias marginais leste e norte do Brasil. Para isto foram desenvolvidos softwares para a modelagem pelo FEM utilizando-se elementos triangulares em malhas n"o regulares, alØm de considerar variaç"o geogrÆÆica de propriedades físicas da placa. $\bigcirc$ m $\varnothing$ todo $\varnothing$ aplicado ao caso das cargas sedimentares do Cone do Amazonas observando-se a influÓncia das cargas de sedimentos no padr"o de flexura nas regiões adjacentes ao Cone. O mØtodo poder $Æ$ ser aplicado tambØm em processos dinâmicos, analisando como ocorreu a migraç"o das ombreiras flexurais durante o processo deposicional. Outras bacias sedimentares ao longo da costa brasileira ser"o analisadas pelo mesmo mØtodo, verificando qual foi o papel da flexura da placa na história de deposiç“o de sedimentos na bacia.

\section{Introduç“o}

A anÆHise de bacias $\varnothing$ a etapa fundamental na avaliaç"o do potencial petrolífero de uma ÆErea. A compreens"o da distribuiç"o e da evoluç"o das seqüOE ncias deposicionais e suas f/Æieies permitem que sejam feitas previsões racionais e realistas sobre a localizaç"o das rochas fonte, rochas reservatório e rochas retentoras de petróleo. A localizaç"o e a forma das principais seqüOநcias deposicionais dependem dos processos mecânicos que levaram à formaç"o da bacia. O mecanismo de abertura do Atlântico Sul, devido a um estiramento da litosfera, subsidOrncia inicial (fase do rifteamento), seguido de subsidOf́cia tØrmica tem sido utilizado no estudo e anA झse das bacias marginais do Brasil e oeste da 'frica. Entretanto, levando-se em conta que existem diferenças marcantes na história de subsidCE ncia dessas bacias, diversos estudos recentes tOE $\mathrm{m}$ mostrado que modelos (usualmente 2-D) simples n"o conseguem prever todas as observações geológicas. Um dos fatores mais difíceis de ser quantificado $\varnothing$ a subsidCE ncia flexural em trOE $s$ dimensões. Modelagem flexural 2-D tem sido utilizada em bacias do tipo ante-país situadas defronte à frentes de empurr"o como no caso dos sub-Andes. Entretanto, resultados recentes demonstram que a geometria da placa e das cargas bem como a variaç"o lateral das propriedades el/sticas da placa diferem substancialmente dos resultados obtidos utilizando aproximações num Øricas bi-dimensionais e sem variaç"o lateral das propriedades mecânicas da placa. Desta forma, pretende-se resolver numericamente a deformaç"o flexural 3-D ao longo da margem continental atlântica do Brasil. Os resultados a serem obtidos ser"o confrontados com as observações geológicas de bacias marginais brasileiras.

\section{Metodologia}

A partir da equaç"o bi-harmônica da flexura w de uma placa fina

$$
(\mathbf{L} \nabla)^{\mathbf{T}} \mathbf{D L} \nabla w+\Delta \rho g w-q=0
$$

onde

$$
\begin{gathered}
(\mathbf{L} \nabla)=\left[\frac{\partial^{2}}{\partial x^{2}}, \frac{\partial^{2}}{\partial y^{2}}, 2 \frac{\partial^{2}}{\partial x \partial y}\right]^{T} \\
\mathbf{D}=D\left[\begin{array}{ccc}
1 & \nu & 0 \\
\nu & 1 & 0 \\
0 & 0 & (1-\nu) / 2
\end{array}\right]
\end{gathered}
$$

(D $\varnothing$ a rigidez el/E stica da placa, $\operatorname{Dr} \varnothing$ a diferença da densidade entre o manto e o material que preenche a placa flexurada e q $\varnothing$ a carga vertical aplicada sobre a placa), $\varnothing$ possível resolve-la atrav $\varnothing$ s do m $\varnothing$ todo dos elementos finitos (Zienkiewicz, 2000) discretizando a regi"o de interesse $\mathbf{W}$, sob certas condições de contorno $\mathbf{f}_{\mathrm{b}}$, da seguinte forma: $w=\mathrm{Na}$, onde $\mathbf{N}$ representa $\mathrm{um}$ vetor com funções interpoladoras conhecidas e a $\varnothing$ um vetor que contØm parâmetros desconhecidos que representam as incógnitas do problema. Com a discretizaç"o pelo mØtodo dos elementos finitos, o problema restringe-se em resolver um sistema linear

$$
\mathbf{K a}=\mathbf{f}
$$

onde 


$$
\begin{gathered}
\mathbf{K a}=\int_{\Omega}\left(\mathbf{B}^{\mathbf{T}} \mathbf{D B}+\mathbf{N}^{T} \Delta \rho g \mathbf{N}\right) d \Omega \mathbf{a} \\
\mathbf{f}=\int_{\Omega} \mathbf{N}^{\mathbf{T}} q d \Omega+\mathbf{f}_{b} \\
\mathbf{B}=(\mathbf{L} \nabla) \mathbf{N}
\end{gathered}
$$

A topologia utilizada foi a desenvolvida por Specht (1988), com triângulos com tr@E s nós, cada nó com trCE s graus de liberdade $(w, x, y)$.

O software foi desenvolvido em linguagem $C$ utilizandose para resolver sistema linear a decomposiç"o de Cholesky.

A malha triangular foi criada a partir do software DISTMESH, desenvolvida por Per-Olof Persson e Gilbert Strang, disponível no site http://www.csit.fsu.edu/.

\section{Flexura no Cone do Rio Amazonas}

Neste modelo estudou-se o efeito flexural da carga de sedimentos na foz do rio Amazonas (Figura. 1) sobre a placa litosfØrica. Considerou-se um valor de $T_{e}=75 \mathrm{~km}$ na regi“o emersa (Figura. 2) e $\mathrm{T} \quad \mathrm{e}=35 \mathrm{~km}$ na regi“o submersa (plataforma continental e placa oceânica). Para o cone de sedimentos foi assumido uma densidade igual a $2300 \mathrm{~kg} / \mathrm{m} 3$ e espessura m/Exima da carga igual a $2 \mathrm{~km}$ (esta espessura est $/ \mathbb{E}$ relacionada com o pacote de sedimentos que est/E acima da posiç"o de equilíbrio da placa quando n"o sujeita a cargas verticais, assim n"o incluindo o pacote de sedimentos que preenchem a porç"o flexurada).

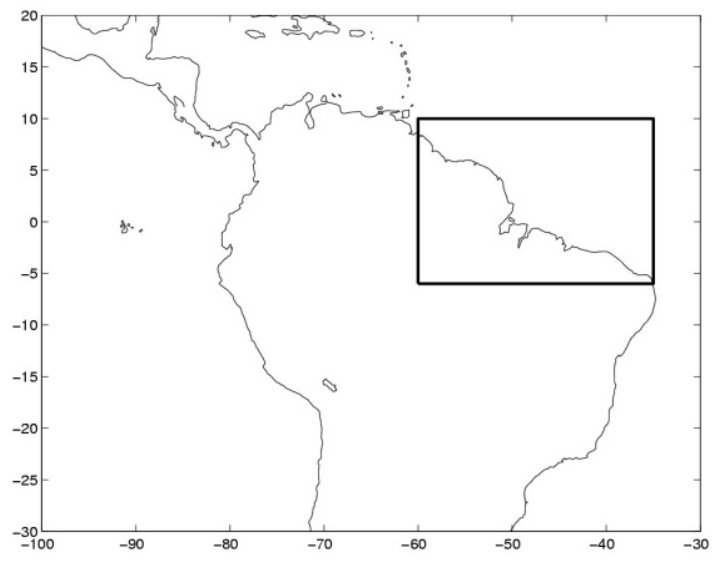

Figura 1. O retângulo delimita a regi“o que foi discretizada para estudar-se o efeito flexural devido a carga de sedimentos do Cone do Amazonas.

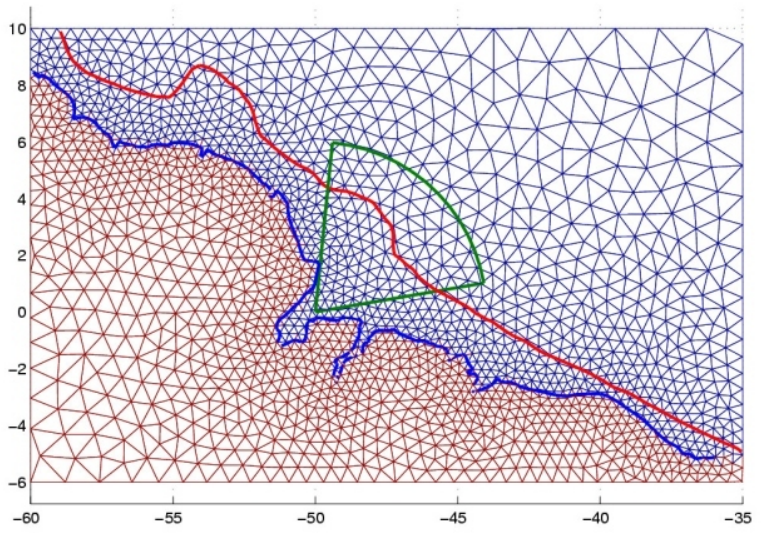

Figura 2. Malha de elementos finitos para a Foz do Rio Amazonas.

\section{Resultados}

Observando-se a Figura 3 vemos que surge uma ombreira flexural na parte emersa na longitude $54^{\circ} \mathrm{W}$ com cerca de $25 \mathrm{~m}$. Esta feiç“o tambØm foi observada por Driscoll \& Karner (1994) e neste trabalho foi sugerido estar relacionado com o Arco de GurupÆ JÆ na plataforma continental existem duas ombreiras flexurais que se dispõem lateralmente ao cone, atingindo amplitudes tambØm entorno de $25 \mathrm{~m}$. Na frente do cone surge uma ombreira flexural com cerca de $50 \mathrm{~m}$ que pode servir de barreira, impedindo que os sedimentos sejam depositados em regiões mais distantes da foz, colaborando para que os sedimentos sejam espalhados lateralmente. Sob a carga de sedimentos, a $\mathrm{m} \mathbb{E} \quad$ xima subsidOfrcia foi de $2.6 \mathrm{~km}$, que somados aos $2 \mathrm{~km}$ de carga resultam em um pacote de sedimentos de $4.6 \mathrm{~km}$.

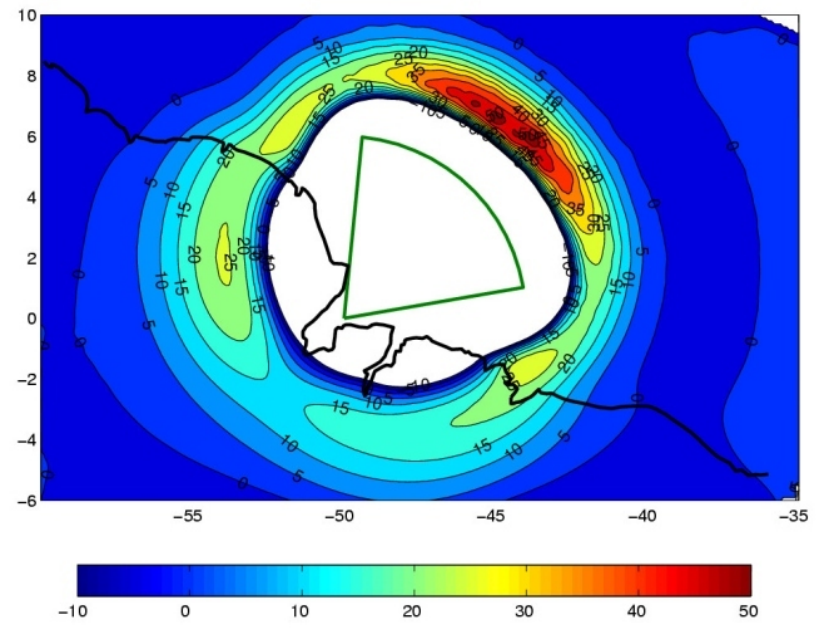

Figura 3. Mapa de deslocamento vertical em metros da placa para o modelo da Figura 2 


\section{Discuss"o e Conclusões}

O mØtodo dos elementos finitos mostrou-se uma ferramenta versÆtil para simulaç"o de flexura de placas. Ao comparar-se os resultados analíticos e os resultados num Øricos para o problema de uma carga pontual, observou-se a convergØEncia do resultado num Ørico para o resultado analítico conforme a malha foi sendo refinada.

Para o caso do Cone do Amazonas, observou-se que, para os valores escolhidos das propriedades físicas da placa litosfØrica e das dimensões e propriedades da carga de sedimentos, a espessura total de sedimentos obtida $(4.6 \mathrm{~km}$ ) est/Epróxima do valor total de sedimentos da Formaç"o Par/E (5-8 km), depositados a partir do meso-Mioceno. $\mathrm{O} \mathrm{m}$ /区imo soerguimento na regi"o emersa atingiu $25 \mathrm{~m}$ e coincide geograficamente com arco de GurupÆE. Assim o efeito flexural do pacote de sedimentos sobre a placa pode estar relacionado com o soerguimento desta regi“o. Foram observados soerguimentos nas regiões laterais ao cone, na plataforma continental, que podem impedir a interconex"o de sedimentos do Cone do Amazonas com bacias adjacentes, isolando-a. J/E na frente do cone houve o maior soerguimento, atingindo cerca de $50 \mathrm{~m}$ de amplitude, representando uma barreira impedindo que os sedimentos sejam depositados em regiões mais distantes da foz.

O mØtodo dos elementos finitos poder $Æ$ ser aplicado tambØm em processos dinâmicos, analisando-se como ocorreu a migraç"o das ombreiras flexurais durante o processo deposicional. Outras bacias sedimentares ao longo da costa brasileira ser"o analisadas pelo mesmo mØtodo, verificando-se qual foi o papel da flexura da placa na história de deposiç“o de sedimentos na bacia.

\section{Agradecimentos}

Ao Programa de Recursos Humanos $n^{\circ} 19$ da AgCEncia Nacional do Petróleo pela bolsa de Iniciaç“o Científica.

\section{ReferŌncias}

Driscoll, N. W., Karner, G. D., 1994, Flexural deformation due to the Amazon Fan loading: A feedback mechanism affecting sediment delivery to margins: Geology, v. 22, pp. 1015-1018.

Specht, B. (1988), Modified shape functions for the three node plate bending element passing the patch test., Int. J. Num. Meth. Eng., 26, 705-715.

Zienkiewicz, O. C., R. L. Taylor (Eds.) (2000), The Finite Element Method, vol. 2, 5th ed., 445 pp., Butterworth Heinemann, Jordan Hill, Oxford. 